\title{
Estudio de Materiales Nanoestructurados por Metalurgia de Polvos dell Ternario Zn-Al-Ag
}

\section{Nancy Badillo Hernández¹, Estela Sarmiento Bustos¹, Arturo Molina Ocampo², and Said Robles Casolco ${ }^{2}$}

${ }^{1}$ Universidad Tecnológica de Emiliano Zapata del Estado de Morelos, Emiliano Zapata, Morelos, México

${ }^{2}$ Universidad Autonóma del Estado de Morelos,Cuernavaca, Morelos, Mexico

\section{Abstract}

In this work one presents the study realized to the compound materials: a) Zn77.0Al22.0-Ag1.0 (wt.\%) [Ternary Alloy], b) Zn77.0-Al22.0-Ag-1.0 (wt.\%) [Compound Material 1] reinforced with $5 \%$ of $\mathrm{NaCl}$, and c) Al77.0-Zn22.0-Ag1.0 (wt.\%)/Si [compound Material 2], the materials were incorporated by means of the alloyed mechanic in a time of grinding of 7 hours. The microstructural characterization

Corresponding Author: Estela Sarmiento Bustos estelasarmiento@ utez.edu.mx

Received: 15 November 2017 Accepted: 5 January 2018 Published: 4 February 2018

Publishing services provided by Knowledge $\mathrm{E}$

(c) Nancy Badillo Hernández et al. This article is distributed under the terms of the Creative Commons Attribution License, which permits unrestricted use and redistribution provided that the original author and source are credited.

Selection and Peer-review under the responsibility of the ESTEC Conference Committee.

G OPEN ACCESS was realized by means of: a) Diffraction of beams-X (DRX), b) Scanning Electronic Microscopy (SEM) and c) Microhardness Vickers (HV). The aim of this work was to study the effect of the microstructure and the hardness of a materials composed bases zinc and his comparison with a ternary alloy. Materials were obtained nanostructure by sizes of crystal in the ternary alloy of $94 \mathrm{~nm}$, and for the compound materials 1 and 2, 116 and $121 \mathrm{~nm}$ respectively. The values of hardness obtained for three systems have direct relation with the size of crystal. The image obtained to SEM at 7 hours of grinding suggests that the ternary alloy presents in the morphology bigger agglomerated particles and the compound material 2 particles small flakes. The results suggest that porous materials were obtained in three studied systems.

Keywords: Mechanical alloy, Metallurgy of powders, Nanocomposite, Reinforcement with particles.

\section{Resumen}

En este trabajo se presenta el estudio realizado a los materiales compuestos: a) Zn77.0Al22.0-Ag-1.0 (wt.\%) [Aleación ternaria], b) Zn77.0-Al22.0-Ag-1.0 (wt.\%) [Material compuesto 1] reforzado con $5 \%$ de $\mathrm{NaCl}$, y c) Al77.0-Zn22.0-Ag-1.0 (wt.\%)/Si [Material compuesto 2], los materiales fueron incorporados mediante el aleado mecánico en un tiempo de molienda de 7 horas. Se realizó la caracterización microestructural mediante: a) Difracción de rayos-X (DRX), b) Microscopía electrónica de barrido (MEB) y c) Microdureza Vickers (HV). El objetivo de este trabajo fue estudiar el efecto de la 
microestructura y la dureza de un material compuesto base zinc y su comparación con una aleación ternaria. Se obtuvieron materiales nanoestructurados con tamaños de cristal en la aleación ternaria de 94nm, y para los materiales compuestos 1 y 2, 116 y $121 \mathrm{~nm}$ respectivamente. Los valores de dureza obtenidos para los tres sistemas tienen relación directa con el tamaño de cristal. El MEB a las 7 horas de molienda sugiere que la aleación ternaria presenta en la morfología partículas aglomeradas y el material compuesto 2 partículas del tipo hojuelas. Los resultados sugieren que se obtuvieron materiales porosos en los tres sistemas estudiados.

Palabras claves: Aleación mecánica, Metalurgia de polvos, Nanocompuesto, Refuerzo con partículas.

\section{1. introducción}

La demanda de nuevas tecnologías requiere procesos de manufactura con nuevos materiales, por una parte y por otra surge la búsqueda de reducción de costos y optimización de los procesos, donde las aleaciones convencionales están siendo sustituidas por materiales compuestos de matriz metálica. Los materiales convencionales presentan tamaños de grano que van desde los micrómetros a cientos de milímetros y contiene cientos de billones de átomos cada uno. Sin embargo, los materiales nanoestructurados son aquellos con una microestructura modulada de cero a tres dimensiones con un tamaño de escala menor de 100 nm (Kear \& Strutt, 1995). La aleación mecánica (AM) es una técnica en estado sólido útil en la fabricación de aleaciones nanocristalinas homogéneas y nanocompuestos que obedece la deformación repetida, la soldadura en frío y el fracturamiento de partículas de polvo (Azimi et al.,2015). Los materiales nanoestructurados se han sintetizado por varias técnicas, una de ellas es, la metalurgia de polvos (MP) y como proceso de fabricación parte de polvos finos, compactación determinada compactado, se calientan en atmósfera controlada (sinterizado) para la obtención de la pieza (Ruiz, et al.,2000). Aleaciones de aluminio se utilizan en diversas aplicaciones como: industria automotriz, marina, y aeroespacial, por alta resistencia específica, ligereza, resistencia a la corrosión y otras características importante para el funcionamiento (Valdez, et al.,2008). El aluminio y sus aleaciones, constituyen uno de los materiales más usados como matriz metálica en la manufactura de materiales compuestos. La ruta de preparación en los materiales compuestos juega un papel importante para la obtención de propiedades mecánicas excelentes como 
dureza, esfuerzo de cedencia y esfuerzo último. Una distribución homogénea de las partículas de refuerzo y un tamaño de grano fino de la matriz contribuyen a mejorar las propiedades mecánicas del material compuesto (Rodríguez, 2017).

\section{Desarrollo Experimental}

\subsection{Materiales}

Se utilizaron los siguientes materiales: Lingote de la aleación Zn-Al-Ag/ Si para la obtención de polvo, Lingote de zinc para la obtención de polvo 99.9\%, Papel aluminio comercial compactado para la obtención de polvos y Polvo de Plata al $99.9 \% \mathrm{NaCl}$ al $99.9 \%$.

\subsection{Equipo}

Se utilizaron los siguientes equipos: Lima Escofina Media Caña Bastarda 10' Truper 15207 y Lima triángulo regular - muza, 8" para la obtención de polvo de zinc. Rayador de acero inoxidable triangular para la obtención de polvo del papel aluminio comercial. Molino planetario de bolas (FRITSCH, Pulverisette 7), con sus accesorios "recipientes y bolas de molienda". Mortero de porcelana para machacar o triturar $\mathrm{NaCl}$. Tamiz de Pruebas Físicas (MONTINOX), Número de malla 60 y 250, con aro y malla de acero inoxidable. Prensa eléctrica digital marca FIICSA. Horno de atmosfera controlada.

\subsection{Método}

En la Figura 1 se presenta un diagrama de flujo de la experimentación. La aleación se hizo en un molino planetario de bolas (FRITSCH, Pulverisette 7) esta técnica puede producir muestras pequeñas ( $1 \mu \mathrm{m}$ de diámetro de las partículas). Se utilizaron bolas de acero inoxidable con un diámetro de 10mm y con una masa de 4.0420 gr para la molienda, en un tiempo 7 horas Para la compresión de los polvos se utilizó una prensa eléctrica digital marca FIICSA y se aplicó una presión de 36.25Tn a cada una de las probetas. La sintetización se llevó a cabo en un horno de atmósfera controlada administrando nitrógeno a una temperatura de $500^{\circ}$ C por 2 horas (tiempo de sinterizado), las probetas se dejaron enfriar a temperatura ambiente. En la Tabla1 se muestran los sistemas de estudio y los materiales base. En la Tabla 1 se muestra la nomenclatura 
empleada de los sistemas estudiados y las características de los materiales base utilizados.

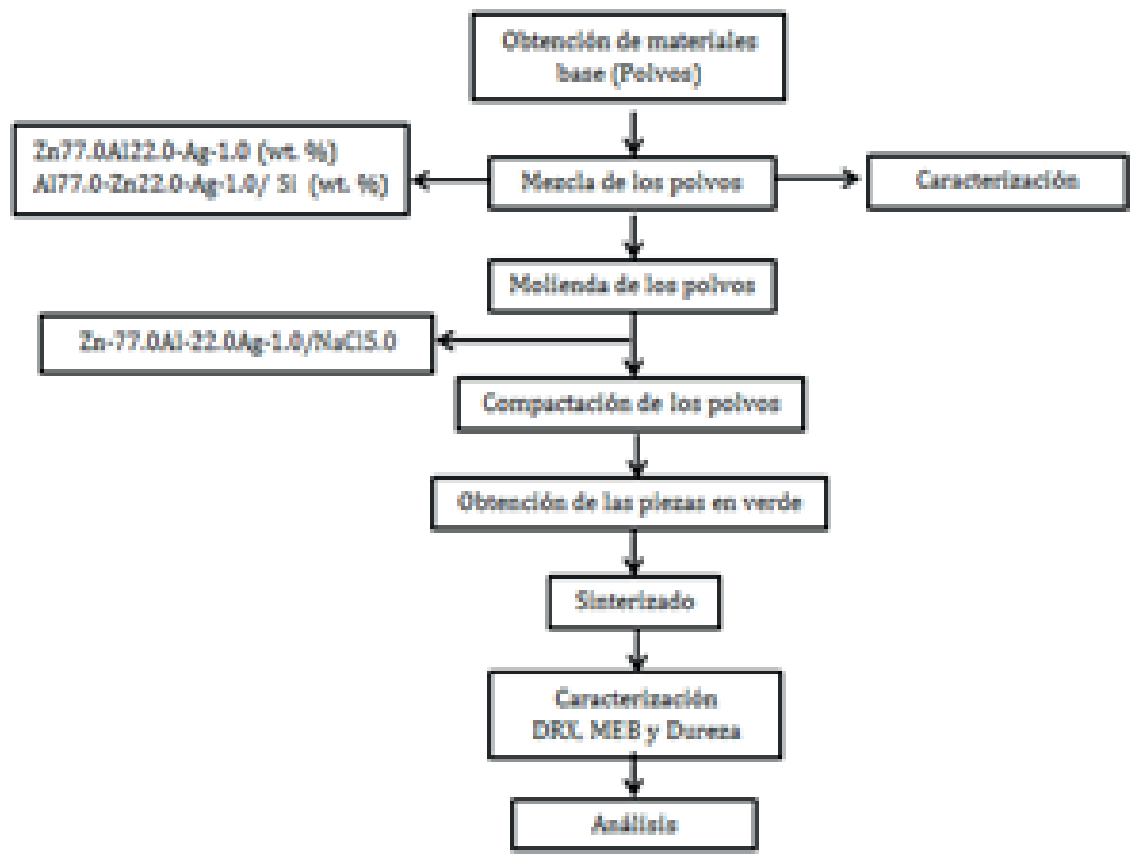

Figura 1: Método Experimental

TABLA 1: Sistema de estudio y materiales base

\begin{tabular}{|c|c|c|c|c|c|}
\hline \multicolumn{3}{|c|}{ Nomenclatura } & \multicolumn{3}{|c|}{ Características } \\
\hline Sistema & Nomenclatura & Composición & $\begin{array}{c}\text { Material } \\
\text { base }\end{array}$ & Pureza (\%) & $\begin{array}{c}\text { Tamaño promedio de } \\
\text { partícula }(\mu \mathrm{m})\end{array}$ \\
\hline Aleación ternaria & Zn-Al-Ag & Zn77.0Al22.0-Ag-1.0 (wt.\%) & $\mathrm{Zn}$ & 99.9 & 65.61 \\
\hline Material compuesto 1 & $\mathrm{Zn}-\mathrm{Al}-\mathrm{Ag} / \mathrm{NaCl}$ & Zn77.0-Al22.0-Ag1.0/NaCl-5.0(wt.\%) & $\mathrm{Al}$ & 98.5 & 186.45 \\
\hline Material compuesto 2 & $\mathrm{Zn}-\mathrm{Al}-\mathrm{Ag} / \mathrm{Si}$ & Al77.0-Zn22.0-Ag1.0/Si (wt.\%) & $\mathrm{Ag}$ & 99.9 & 129.31 \\
\hline
\end{tabular}

\subsection{Caracterización}

\subsubsection{DIFRACCIÓN de rayos $X(D R X)$}

Los difractogramas se obtuvieron con el equipo con el equipo D2 PHASER (30 kV y 10 $\mathrm{mA})$ con radiación Cu-K $\alpha(\lambda=1.54184 \AA$ ). Los patrones de difracción fueron colectados en el rango de $2 \theta$ of $20^{\circ}-80^{\circ}$ con un paso de $0.0101^{\circ}$. Para la identificación de las fases se utilizaron fichas JCPDS. El tamaño del cristal (D) fue determinado a partir del ensanchamiento de picos de DRX utilizando la ecuación de Scherrer (Ec.1):

$$
\mathbf{D}=\frac{\mathbf{k} \lambda}{\beta \operatorname{Cos} \theta}
$$


TABLA 2: Características de los materiales base

\begin{tabular}{|c|c|c|l|}
\hline $\begin{array}{c}\text { Material } \\
\text { base }\end{array}$ & $\begin{array}{c}\text { Pureza } \\
(\%)\end{array}$ & $\begin{array}{c}\text { Tamaño } \\
\text { promedio de } \\
\text { partícula } \\
(\mu \mathrm{\mu m})\end{array}$ & Morfología \\
\hline $\mathrm{Zn}$ & 99.9 & 65.61 & Irregular \\
\hline $\mathrm{Al}$ & 98.5 & 186.45 & Irregular \\
\hline $\mathrm{Ag}$ & 99.9 & 129.31 & Irregular \\
\hline
\end{tabular}

Dónde: D es el tamaño de cristal, $k$ es una constante de proporcionalidad (0.9), $\lambda$ es la longitud de onda de rayos $X, \beta$ es la anchura a media altura del pico de difracción y $\theta$ es el ángulo de difracción de Bragg. Se determinó el tamaño del cristal para el plano de difracción CCC- Zn (101). El parámetro de red se determinó para el plano de difracción CCC-Zn (101) fue calculado utilizando la relación.

\subsubsection{Microscopía electrónica de BARRIDO (MEB)}

Se utilizó un microscopio LEO 1450 VP, operado a $15 \mathrm{Kv}$. El equipo cuenta con un sistema de análisis por Espectroscopía de energía dispersiva (EDX). Las muestras fueron desbastadas con papel abrasivo de SiC del No. 600 hasta el 2000 para proporcionar una superficie plana se utilizó en una pulidora Marca LECO SPECTRUM SYSTEM 1000, y fueron pulidas con $\mathrm{Al}_{2} \mathrm{O}_{3}$ en agua $0.05 \mu \mathrm{m}$ hasta obtener acabado espejo. Se obtuvieron los tamaños de partícula de los materiales base y los tamaños de poro de la aleación ternaria y de los materiales compuestos 1 y 2 , mediante el programa Imagej.

\subsubsection{Dureza Vikers}

La dureza en escala Vickers se determinó con un microdurómetro marca Matzuzawa modelo MHT2, de acuerdo a la norma ASTM E92.

\section{RESULTADOS}

\subsection{Caracterización de los materiales base}

Los materiales base (Zn-Al-Ag) se caracterizaron determinando las propiedades: pureza, tamaño promedio de partícula y morfología. Las características de los polvos de origen, se muestran en la Tabla 2. 


\subsection{Difracción de rayos $X(D R X)$}

En la figura 2 se muestran los difractogramas obtenidos de la aleación ternaria ZnAl-Ag y de los materiales compuestos. Las fases presente en el patrón de difracción mostrado en la Figura.2 comparan los diferentes métodos de obtención para cada uno de los elementos compuestos, para el caso 1a, se tiene una aleación por medio fusión tradicional, donde las condiciones de atmosfera controlada son causantes de la baja concentración de los elementos de bajo punto de fusión, por lo cual a concentración de la fase $(\beta)$ donde representa al componente de $Z n$, con una disminución en la composición, sin embargo en la Figura. 2 (1b), demuestra que el procedimiento de metalurgia de polvos de acuerdo al Figura.1 la composición y el procedimiento se encuentra en condiciones óptimas de acuerdo a la aleación maestra de análisis, sin embargo, comparativamente, en el proceso de la adición de componentes adicionales a la aleación maestra la integración de los elementos se difunden por lo cual la intensidad de los elementos disminuye mostrando una difusión en los elementos adicionados por la aleación mecánica y el proceso de sinterizado. El pico con mayor intensidad es el de Zn, en la aleación ternaria, el cual difracta a $2 ?=43.22$. Los tamaños de cristal para las aleaciones promedio fue de $94 \mathrm{~nm}$. Los tamaños de cristal obtenidos del sistema Zn77.0 Al-22.0 Ag-1.0/ $\mathrm{NaCl}$ y Al-77.0 Zn-22.0 Ag-1.0/Si, fueron 43.83 y 46.76, respectivamente (Ver Tabla 3).

\subsection{Microscopía electrónica de barrido (MEB)}

En la Figura 3(a,b y c) se muestra la morfología de los componentes en condiciones mecánicas de obtención por limadura, por lo que se observa que se tiene inicalmente una microestrucrura de hojuela. En la figura zb se puede distinguir que el material de Al, tiene un tamaño mayor a las 85 micras y que mayoritariamente dadas las condiciones de material de reciclado la obtencion y separacion se obtienen estas caracteristicas de tamaño porcentual. En la figuras za y b se tiene un promedio general de acuerdo a las consideraciones de el cribado utilizado en la obtención de separacion y condiciones similares.

En la Figura 4 se muestra la morfología obtenida por MEB a las 7 horas de molienda, comparativamente en la microestructura 4 a, las condiciones de molienda y granulometría se identifica un patrón mayoritariamente homogéneo de condensación para los tres componentes, en contraposición, la figura 4 b muestra laminas con de los componentes adicionados donde la molienda no favorece a la compactación e integración 


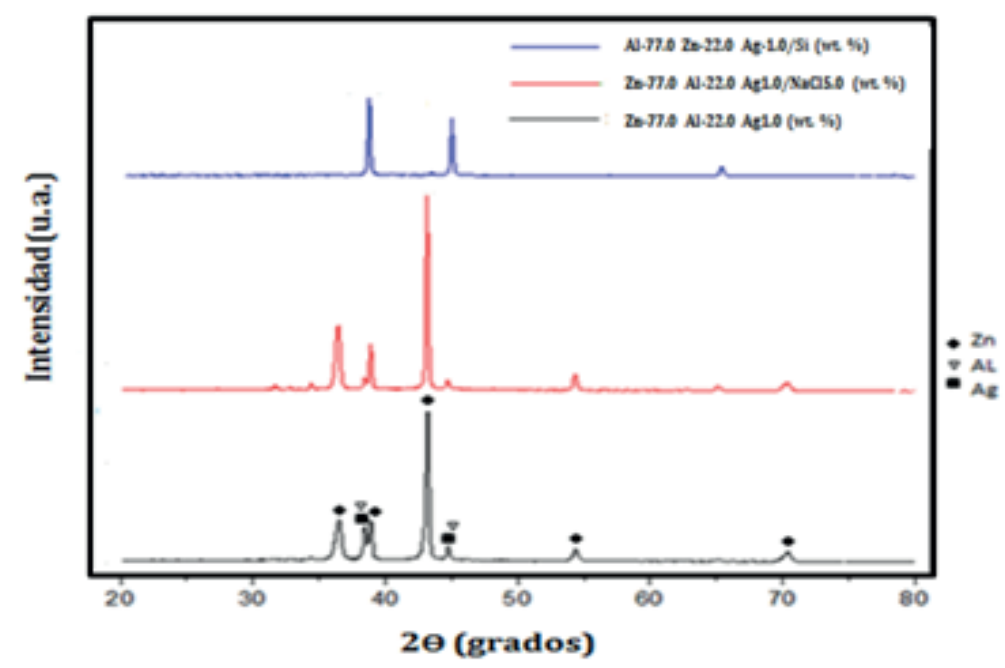

Figura 2: DRX del ternario Zn-Al-Ag sinterizadas a $500^{\circ}$
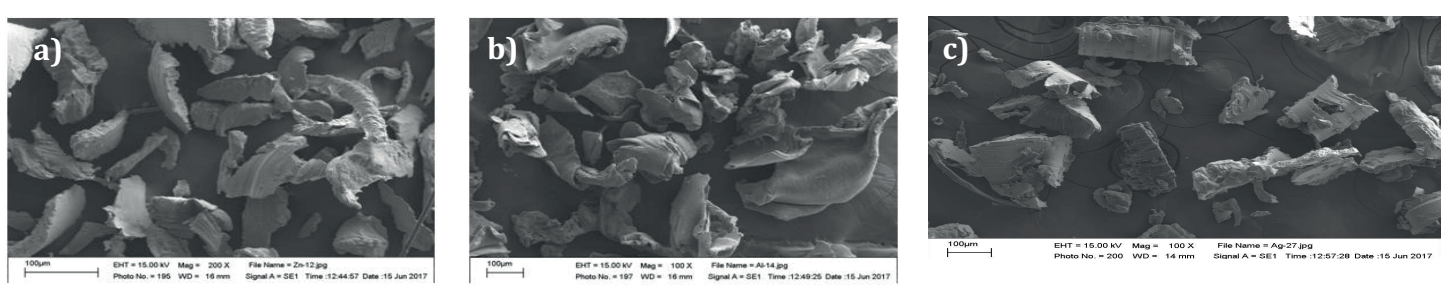

Figura 3: Morfología de los materiales base: Magnificación: a) Zn 200X, b)Al 100X y c) Ag 100X.

homogénea de los elementos. En la Figura 5 se muestra la morfología obtenida por SEM, posterior al sinterizado. Se observa que la aleación ternaria (a), correspondiente a una integración completa, pero con características de rechupe por atrapamiento de oxígeno en el proceso, donde se muestra una correcta consolidación del sinterizado efecto de la temperatura adecuada en el proceso. En la figura 5 b y $5 c$ se muestra la presencia de los precipitados donde no se permite las condiciones de integración de los elementos adicionados de acuerdo a la Tabla 1 de condiciones de integración en la aleación base.
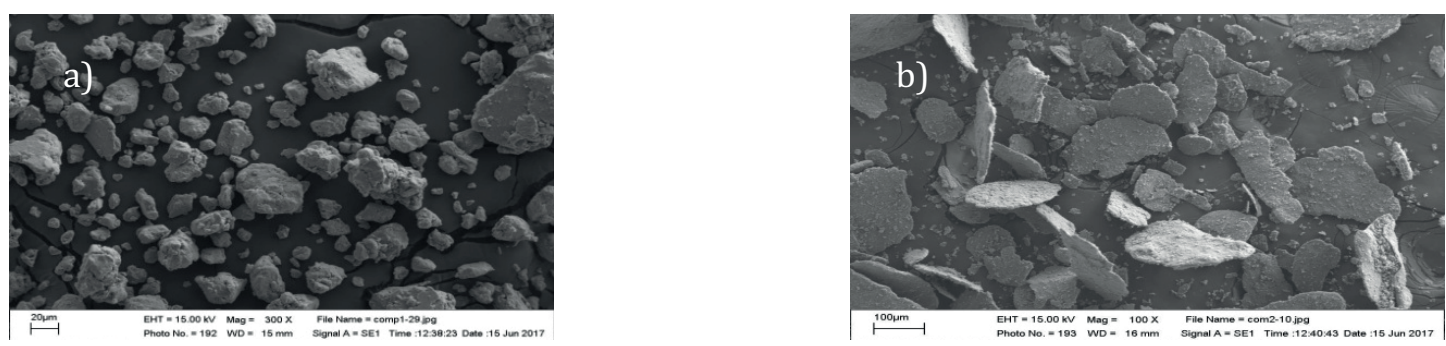

Figura 4: Morfología de los materiales compuestos con 7 horas de molienda: a) Zn-77.0 Al-22.0 Ag1.0 300X [aleación ternaria] y b) Al-77.0 Zn-22.0 Ag-1.0/Si - 100X [Material compuesto 2]. 
TABLA 3: Tamaños de partícula, poro y cristal de la aleación ternaria y de los materiales compuestos 1 y 2.

\begin{tabular}{|c|c|c|c|}
\hline Sistema & $\begin{array}{c}\text { Tamaño de } \\
\text { partícula ( } \mu \mathrm{m}) \\
\text { (ImagenJ) }\end{array}$ & $\begin{array}{c}\text { Tamaño de } \\
\text { poro } \\
(\mu \mathrm{m})(\text { ImagenJ) }\end{array}$ & $\begin{array}{c}\text { Tamaño de } \\
\text { cristal (nm) } \\
\text { (DRX) }\end{array}$ \\
\hline $\begin{array}{c}\text { Aleación } \\
\text { ternaria }\end{array}$ & 17.23 & 2.001 & 94.87 \\
\hline $\begin{array}{c}\text { Material } \\
\text { Comp } 1\end{array}$ & 17.23 & 2.32 & 116.68 \\
\hline $\begin{array}{c}\text { Material } \\
\text { Comp } 2\end{array}$ & 83.94 & 1.23 & 121.48 \\
\hline
\end{tabular}

\subsection{Dureza Vikers}

En la Tabla 4 se muestran los valores promedio de Dureza Vikers de la aleación ternaria y de los materiales compuestos 1 y 2, Zn-77.0 Al-22.0 Ag-1.0/ $\mathrm{NaCl}$ y Al-77.0 Zn-22.0 $\mathrm{Ag-1.0/Si,} \mathrm{respectivamente.} \mathrm{Las} \mathrm{condiciones} \mathrm{de} \mathrm{aumento} \mathrm{en} \mathrm{los} \mathrm{valores} \mathrm{como} \mathrm{se} \mathrm{mues-}$ tran en la figura 5 b y $5 c$ se encuentra precipitados que realizan que el mecanismo de endurecimiento sea mayor.
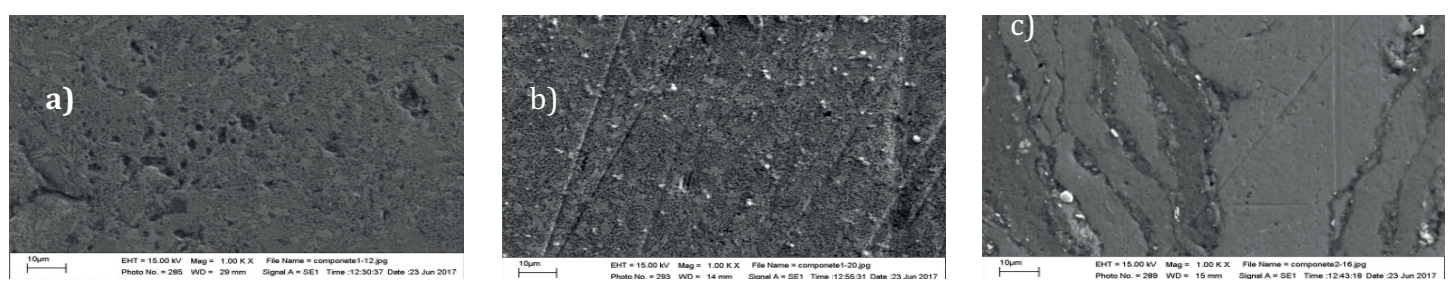

Figura 5: Micrografías de la aleación ternaria y de los materiales compuestos 1 y 2 después del sinterizado: a) Aleación ternaria Zn-77.0Al-22Ag-1.0 a 1000X, b) Zn-77.0 Al-22.0 Ag1.0/NaCl [Material compuesto 1] a 1000X y c) Al-77.0 Zn-22.0 Ag1.0/Si [Material compuesto 2] a 1000X.

\section{Discusión}

Los difractogramas de rayos $X$ mostraron un refinamiento en el tamaño del cristal de 94 nm para el plano Zn (101) que corresponde al pico de mayor intensidad de la aleación ternaria después de las 7 h de molienda. Los materiales obtenidos sugieren un comportamiento típico de materiales procesados mediante aleado mecánico; se observa un incremento en el ancho medio del pico y desplazamiento de los perfiles de difracción, además de que desvanecimiento de la principal línea de difracción. El material compuesto Zn-Al-Ag/ Si obtuvo el mayor valor de dureza de $46.76 \mathrm{HV}$, y la aleación ternaria presentó el menor valor de dureza HV de 23.94. Trabajos previos (Casolco et al., 2004) y (Rodríguez, 2017) reportan la implementación del material Zn, se comparó la dureza de los materiales compuestos y se observa que el uso del aluminio comercial disminuye el valor de dureza (HV). Los parámetros que influyen en la fabricación de 
las probetas son la presión, la temperatura y el tiempo de sinterización. Los materiales compuestos estudiados presentan características morfológicas de porosidad. Con base en lo anterior el Aleado Mecánico (AM) es un excelente método para obtener materiales nanoestructurados homogéneos con buenas propiedades mecánicas.

\section{Conclusiones}

Se obtuvo un material nanoestructurado mediante el aleado mecánico (AM) los resultados obtenidos sugieren que puede tener aplicaciones para la elaboración de filtros de gases en equipos de laboratorio. Dentro del análisis mecánico se puede observar que el incremento de los aleantes para el material testigo, permite identificar que existe un mecanismo de precipitación en función de las condiciones de los elementos, el cual se refleja en el comportamiento de la dureza, lo cual es un atributo importante para poder continuar realizando estudios más extensivos y poder encontrar parámetros y condiciones para explicar la fenomenología de la presente línea de investigación.

\section{Agradecimientos}

A la Dra, S. Valdez- ICF-UNAM, al CIICAp-UAEM, al CONACyT (Concejo Nacional de Ciencia y Tecnología) y la Universidad Tecnologica de Emiliano Zapata del Estado de Morelos (UTEZ), S.N.I. -CONACYT.

\section{Referencias}

[1] Kear, B. H., \& Strutt, P. R. (1995). Chemical processing and applications for nanostructured materials. Nanostructured materials, 6(1), 227-236.

[2] Azimi, A., Shokuhfar, A., \& Nejadseyfi, O. (2015). Mechanically alloyed Al7075-TiC nanocomposite: Powder processing, consolidation and mechanical strength. Materials \& Design (1980-2015), 66, 137-141.

[3] Ruiz-Navas, E. M., da Costa, C. E., López, F. V., \& Castelló, J. M. T. (2000). Aleación mecánica: Método de obtención de polvos metálicos y de materiales compuestos. Revista de Metalurgia, 36(4), 279-286.

[4] Valdez, S., Campillo, B., Pérez, R., \& Martínez, L. (2008). Synthesis and microstructural characterization of Al-Mg alloy-SiC particle composite. Materials letters, 62(17), $2623-2625$. 
[5] Fathy, A., Wagih, A., El-Hamid, M. A., \& Hassan, A. A. (2014). The effect of Mg adds on morphology and mechanical properties of Al-xMg/10Al 203 nanocomposite produced by mechanical alloying. Advanced Powder Technology, 25(4), 1345-1350.

[6] Rodríguez P.O (2017). Síntesis y caracterización microestructural de un material compuesto Al-Mg-Zn-Si- $\mathrm{Y}_{2} \mathrm{O}_{3}$ (Tesis de doctorado en Ingeniería y Ciencias Aplicadas). UAEM, Cuernavaca, Morelos.

[7] Robles C.S (2004). Caracterización mecánica del eutectoide Zn-Al, modificado con plata. (Tesis por optar por el grado de doctor en ciencias). UNAM, México Distrito Federal.

\section{Authorization and Disclaimer}

Authors authorize ESTEC to publish the paper in the conference proceedings. Neither ESTEC nor the editors are responsible either for the content or for the implications of what is expressed in the paper. 